\title{
Association of Venous Thromboembolism and Early Mortality in Patients with Newly Diagnosed Metastatic Non-Small Cell Lung Cancer
}

\author{
Yanping Su $\mathbb{D}^{\prime}$ \\ Meirong Huo' \\ Lin $\mathrm{Hua}^{2}$ \\ Yuan Zhang' \\ Jiawen $\mathrm{Yi}^{1}$ \\ Shu Zhang' \\ Jie $\mathrm{Li}^{\prime}$ \\ Yuhui Zhang' \\ 'Department of Respiratory and Critical \\ Care Medicine, Beijing Chao-Yang \\ Hospital, Capital Medical University, \\ Beijing Institute of Respiratory Medicine, \\ Beijing, People's Republic of China; \\ ${ }^{2}$ School of Biomedical Engineering, \\ Capital Medical University, Beijing, \\ 100069, People's Republic of China
}

Correspondence: Yuhui Zhang Department of Respiratory and Critical Care Medicine, Beijing Chao-Yang Hospital, Capital Medical University, Beijing Institute of Respiratory Medicine, 8 Gongtinan Road, Chaoyang District, Beijing, People's Republic of China Tel +86 13520208369

Email zhangyhcy@163.com
Purpose: To explore the relationship between venous thromboembolism (VTE) and early mortality (within six months) in Chinese patients with newly diagnosed metastatic non-small cell lung cancer (NSCLC) after entering the era of precision treatment.

Methods: A cohort of 706 consecutive subjects with newly diagnosed metastatic NSCLC were prospectively observed. Clinical and survival data were recorded over a six-month follow-up period. The predictive factors for the occurrence of VTE and the relationship with early mortality were evaluated through univariate and multivariate analyses.

Results: During the six-month follow-up period, VTE events occurred in $12.2 \%(86 / 706)$ of the enrolled patients. In the multivariate analyses for VTE, an age older than 70 years (vs < 70: sub-distribution hazard radio [SHR], 1.678; 95\% confidence interval (CI), 1.073-2.600; $P=0.022$ ), an Eastern Cooperative Oncology Group performance status $\geq 2$ (vs 0/1: SHR, 1.946; 95\% CI, 1.277-2.970; $P=0.002$ ), and having an $A L K$ rearrangement (vs nonrearrangement: SHR, 2.377; 95\% CI, 1.186-4.760; $P=0.015)$ were significantly associated with the occurrence of VTE. Within six months, 116 subjects $(16.4 \%)$ died, and the occurrence of VTE (vs no VTE: adjusted HR: 1.863; 95\% CI: 1.178-2.947, $P=0.008$ ) was remarkably associated with early mortality. Further analysis showed 98 patients $(13.9 \%)$ with early mortality had $E G F R / A L K$ wild-type genes, with a risk of early mortality 5.935 -fold higher than that of patients with an $E G F R$ mutation/ALK rearrangement. Finally, subgroup analyses showed that VTE occurrence was a significant factor for predicting early mortality in patients with $E G F R / A L K$ wild-type genes (adjusted HR: 1.682; 95\% CI: 1.023-2.768, $P=0.041$ ).

Conclusion: Patients with an EGFR mutation/ALK rearrangement had a significantly decreased risk of early mortality in the era of targeted therapy; however, VTE occurrence remained an important predictor for early mortality in metastatic NSCLC patients, especially in patients with $E G F R / A L K$ wild-type genes.

Keywords: venous thromboembolism, non-small cell lung cancer, early mortality

\section{Introduction}

Venous thromboembolism (VTE) can be a fatal complication of malignant tumors and is the second leading cause of death after that associated with the malignant tumor itself. ${ }^{1,2}$ The risk of VTE occurrence in patients with cancer is approximately 4- to 6.5-fold higher than in those without cancer, ${ }^{3,4}$ and the risk is highest in the first six months after cancer diagnosis. ${ }^{5}$ Lung cancer is associated with a high risk of occurrence of VTE. Our previous multicenter study showed a high prevalence of VTEs in patients with newly diagnosed lung cancer, especially metastatic non-small 
cell lung cancer (NSCLC). ${ }^{6}$ Thereafter, assessments of our own cohorts and other studies have also revealed an association between driver oncogene alterations and VTE occurrence in NSCLC patients. ${ }^{7-10}$

The rates of early mortality vary greatly based on the cancer type, stage, and treatment, although the rate is especially high in patients with advanced cancer, ${ }^{11}$ and approximately $60 \%$ of all newly diagnosed NSCLC patients have advanced disease. ${ }^{12}$ Molecular testing has become a standard procedure in patients with metastatic NSCLC, and targeted agents for various genetic alterations have led to significant improvements in survival. ${ }^{13,14}$ Previous studies have shown that cancer patients with VTE experience a greater risk of early all-cause mortality than those without VTE. ${ }^{15,16}$ However, in the modern era of molecular targeted therapy, the association between the occurrence of VTE and early mortality in metastatic NSCLC remains underdefined. Therefore, we conducted a prospective, observational, cohort study in consecutive patients with metastatic NSCLC to explore the association between the occurrence of VTE and early mortality within six months in a real-world clinical practice environment after entering the era of precision treatment.

\section{Materials and Methods}

\section{Study Population}

The study was conducted at Beijing Chao-Yang Hospital, Capital Medical University. Patients who were newly diagnosed with NSCLC between January 2014 and December 2019 were consecutively enrolled. The inclusion criteria were as follows: confirmation of NSCLC by cytological or histological examination; stage IV Tumor Node Metastasis (TNM) classification; ${ }^{17}$ identification of epidermal growth factor receptor $(E G F R)$, Kirsten rat sarcoma (KRAS) mutations, and anaplastic lymphoma kinase $(A L K)$ alterations; and voluntary participation. This cohort excluded patients who had a history of VTE (diagnosed more than three months before enrollment) and continuous anticoagulant drug use. Patients in this cohort were observed prospectively from the time of diagnosis to six months later, unless a patient voluntarily withdrew from the study, was lost to follow-up, or death occurred. All subjects provided written informed consent. The Ethics Committees of Beijing Chao-Yang Hospital, Capital Medical University (No. 2009-4 and 2016-79) provided approval to conduct this prospective cohort study. ${ }^{9,10}$ This study conformed with the tenets of the Declaration of Helsinki.

\section{Data Collection}

Clinical and examination data were recorded from the patients' medical records. Survival data were collected from the patients' 2-, 4-, and 6-month follow-up calls and outpatient visits. Finally, the following variables were recorded for each subject for group comparisons: age ( $<70$ vs $\geq 70$ years); sex (male vs female); Eastern Cooperative Oncology Group (ECOG) performance status (PS) (0-1 vs $\geq 2$ ); history of smoking (current/former vs never); histology type (adenocarcinoma vs squamous cell carcinoma vs other NSCLC); body mass index (BMI) $\left(<25 \mathrm{vs} \geq 25 \mathrm{~kg} / \mathrm{m}^{2}\right)$; EGFR (mutated vs wild-type), KRAS (mutated vs wild-type), and $A L K$ (rearrangement vs non-rearrangement) genotypes; intracranial metastasis (yes vs no); VTE status; VTE time; and early mortality during the six-month follow-up period.

\section{Driver Oncogene Evaluation}

$E G F R$ and KRAS mutations were examined via amplification refractory mutation system polymerase chain reaction (ARMS-PCR). $A L K$ rearrangements were confirmed by fluorescence in situ hybridization. Detailed information pertaining to these evaluation techniques has been provided in our previous publications. ${ }^{9,10}$

\section{Diagnosis of VTE}

Symptomatic patients underwent imaging examinations to confirm the occurrence of VTE events (venous ultrasound imaging or computed tomography (CT) venous angiography for deep vein thrombosis (DVT); CT pulmonary angiography or ventilation/perfusion scanning for pulmonary embolism). All VTE events were finally submitted to a panel of vascular and radiologic experts for review. All incidentally detected VTE cases were included. The panel determined if an incidental VTE event was of clinical significance. The VTE time was recorded from the date of the first objective diagnosis of metastatic NSCLC to either the date of the VTE occurrence or the last medical follow-up record, whichever occurred earlier.

\section{Diagnosis of Early Mortality}

Survival time was recorded from the day of first diagnosis of metastatic NSCLC to either the day of death or loss to follow-up. Subjects who died within six months after diagnosis were assigned to the early mortality group. 


\section{Statistical Analysis}

All variables were considered to be categorical and are described by frequencies and percentages. In the univariate analysis for VTE, Gray's test was performed to compare variables between subgroups of patients with and without VTE. A death/withdrawal competing risk model was employed to determine the independent variables for the multivariate analyses. In the multivariate analysis, the included variables were those with statistical significance $(P<0.05)$ in the univariate analysis. The cumulative incidence of early mortality was analyzed using KaplanMeier survival curves, and differences between the groups were evaluated through Log rank testing. Univariate and multivariate Cox proportional hazards regression analyses were used to identify the independent predictors of early mortality. All statistical tests were two-tailed, and a $P$-value $<0.05$ was considered statistically significant. R 4.0.2 software (R Foundation, Vienna, Austria) was used to conduct Gray's test and the Fine-Gray regression analysis. Cox proportional hazards regression analyses were performed using Statistical Package for the Social Sciences (SPSS) 24.0 (IBM, Armonk, NY, USA). An epidemiologist reviewed the study.

\section{Results}

\section{Population}

In total, 718 patients with newly diagnosed stage IV NSCLC were recruited for this study. Overall, seven patients were excluded due to a history of VTE spanning longer than three months before enrollment, and five patients were excluded due to the continuous administration of anticoagulant drugs. Finally, 706 subjects with metastatic NSCLC were included in the present cohort (Figure 1) and the analysis.

Of these patients, $83 \%(n=586)$ had been diagnosed with adenocarcinoma. The median age of all the patients at diagnosis was 64 years, and $73.1 \%(n=516)$ of patients were younger than 70 years of age. In terms of sex, $55.0 \%$ $(n=388)$ were male, and $45.3 \%(n=320)$ were former or current smokers. Furthermore, $23.8 \%(n=168)$ of patients exhibited a poor ECOG PS, and only $30.9 \%(n=218)$ had a BMI $\geq 25 \mathrm{~kg} / \mathrm{m}^{2}$. The identified driver oncogene types included mutations of EGFR $(43.3 \%, \mathrm{n}=306)$ and $K R A S$ $(11.3 \%, \mathrm{n}=80)$, and $A L K$ rearrangements $(5.1 \%, \mathrm{n}=36)$. Intracranial metastasis was noted in $14.4 \%(\mathrm{n}=102)$ of patients at diagnosis. Treatments administered during the observation period included targeted therapy with tyrosine kinase inhibitors (TKIs) $(40.2 \%, \mathrm{n}=284)$, chemotherapy $(37.8 \%, \mathrm{n}=267)$, and other types of anticancer therapy (immunotherapy, radiotherapy, palliative surgery, and palliative support treatment) $(22.0 \%, \mathrm{n}=155)$ (Table 1).

\section{Development of VTE and Early Mortality}

Overall, 86 patients developed VTE (12.2\%), 10.4\% (n=9) of whom experienced a pulmonary embolism (PE), 68.6\% $(n=59)$ of whom had DVT (including upper extremity, lower extremity, or neck DVT), and $20.9 \%(n=18)$ of whom experienced both PE and DVT. Most patients received low-molecular-weight heparin and/or warfarin or rivaroxaban anticoagulant therapy, although seven patients did not receive anticoagulant therapy due to contraindications. Hematuria occurred in one patient, and one patient experienced hemoptysis, although both recovered quickly after discontinuing the administration of anticoagulant agents. No major bleeding events occurred.

The proportion of patients who experienced early mortality during the six-month follow-up period was $16.4 \%$ $(n=116)$. At the time of the final analysis for VTE, $18.7 \%$ of patients $(n=132)$ had experienced death from a competing event or had withdrawn from the study. The baseline characteristics and the treatments administered during observation days are provided in Table 1 .

\section{Risk Factors Associated with VTE}

In the univariate analysis, the occurrence of VTE was higher in patients older than 70 years of age (Gray's test $P=0.024$ ) and in patients with an ECOG PS $\geq 2$ (Gray's test $P<0.001)$. $A L K$ alterations were also significantly associated with the occurrence of VTE (Gray's test, $P=$ $0.014)$, whereas $E G F R$ mutations were negatively associated with VTE occurrence (Gray's test, $P=0.018$ ), and there was no significant association between other clinical features/driver oncogene alterations and the occurrence of VTE (Table 2).

Subsequently, Fine-Gray regression analysis was conducted to identify the possible risk factors for VTE occurrence, including age, ECOG PS, the presence of EGFR alterations, and $A L K$ status. Ultimately, an age older than 70 years (vs $<70$ years: sub-distribution hazard radio [SHR], 1.678; 95\% confidence interval (CI), 1.073-2.600; $P=0.022$ ), an ECOG PS $\geq 2$ (vs 0/1: SHR, 1.946; 95\% CI, $1.277-2.970 ; P=0.002)$, and the presence of an $A L K$ rearrangement (vs non-rearrangement: SHR, 2.377; 95\% CI, $1.186-4.760 ; P=0.015)$ were identified as independent risk factors for VTE occurrence (Table 2). 


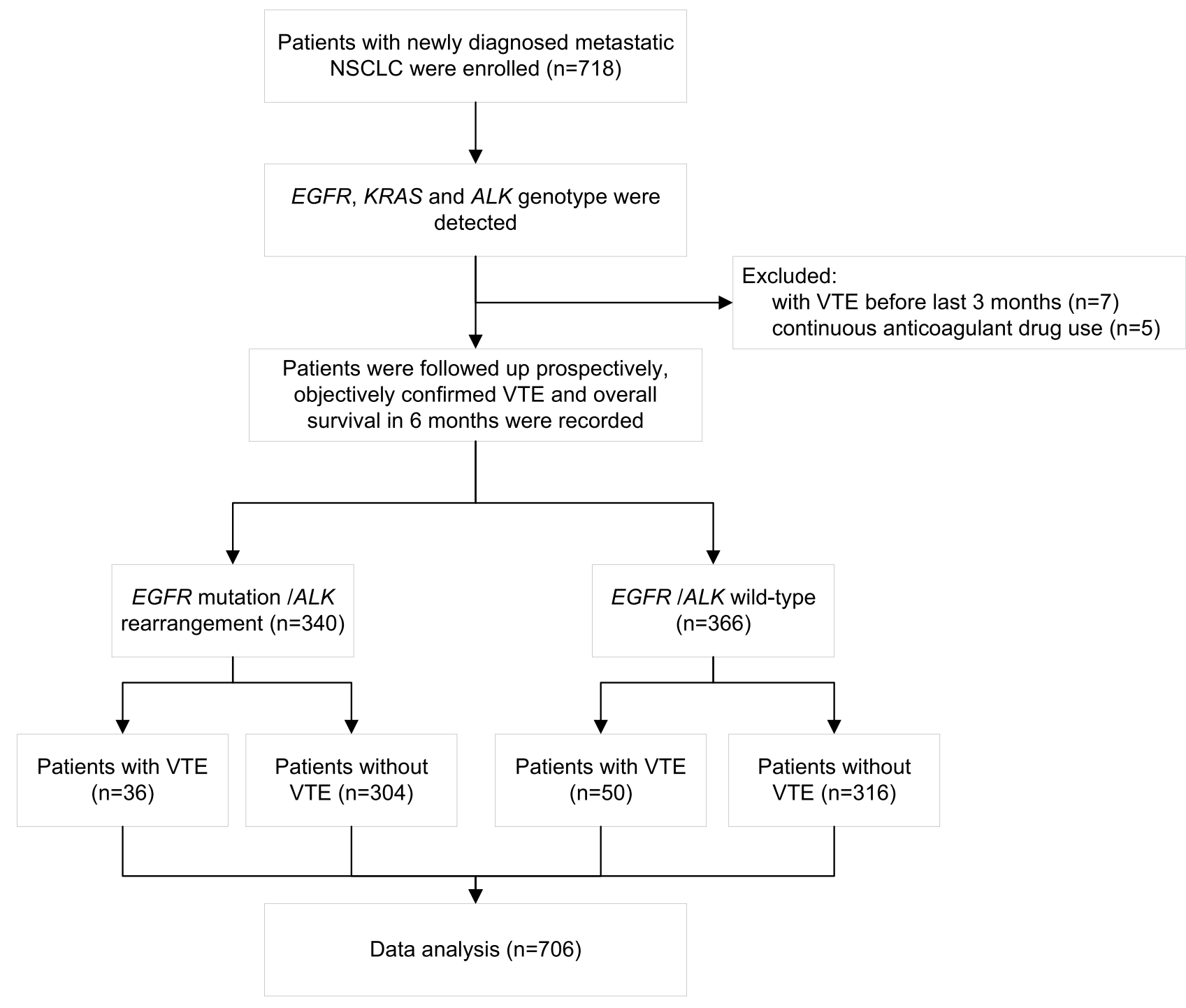

Figure I Study flow diagram.

Abbreviations: NSCLC, non-small cell lung cancer; ALK, anaplastic lymphoma kinase; EGFR, epidermal growth factor receptor; KRAS, Kirsten rat sarcoma; VTE, venous thromboembolism.

\section{Association of VTE with Early Mortality}

The Kaplan-Meier survival analyses showed that the risk of early mortality was significantly higher in the VTE group (vs no VTE: $P=0.001$ ), and the multivariate $C o x$ analysis (adjusted for sex, age, BMI, intracranial metastasis, tumor histology, and driver oncogene type) also confirmed that VTE (adjusted HR: 1.863; 95\% CI: 1.178-2.947, $P=0.008$ ) was an independent risk factor for early mortality (Figure 2A). Further analysis showed that 98 patients who experienced early mortality had $E G F R / A L K$ wild-type genes, and their risk of early mortality was 5.935 -fold higher than that of patients with an $E G F R$ mutation/ALK rearrangement (Figure 2B). In addition, subgroup analyses were performed according to driver oncogene alterations, which showed significant differences in the rate of early mortality among the driver oncogene groups with different VTE statuses based on the Log rank tests $(P<0.0001)$. More specifically, the group with $E G F R / A L K$ wild-type genes who experienced VTE displayed the poorest survival outcomes (Figure 2C). Finally, after adjusting for sex, age, BMI, intracranial metastasis, tumor histology, and treatment method, the occurrence of VTE was a significant factor for predicting early mortality only in patients with $E G F R / A L K$ wild-type genes (adjusted HR: 1.682; 95\% CI: 1.023-2.768, $P=0.041$ ) (Table 3).

\section{Discussion}

In this real-world cohort study of Chinese patients with metastatic NSCLC, we examined the predictive factors for 
Table I Patient Demographics and Clinical Characteristics and Primary Study Outcomes

\begin{tabular}{|c|c|c|c|}
\hline Characteristic & $\begin{array}{l}\text { Patients } \\
\text { n (\%) }\end{array}$ & $\begin{array}{c}\text { Patients with VTE } \\
\text { n (\%) }\end{array}$ & $\begin{array}{c}\text { Patients with Early Mortality } \\
\text { n (\%) }\end{array}$ \\
\hline Overall & $706(100)$ & $86(12.2)$ & $116(16.4)$ \\
\hline Age (years) & 64 & 65 & 67 \\
\hline 25th-75th percentile & $56-70$ & $54.8-72$ & $57-73$ \\
\hline$<70$ & $516(73.1)$ & $54(10.5)$ & $70(13.6)$ \\
\hline$\geq 70$ & $190(26.9)$ & $32(16.8)$ & $46(24.2)$ \\
\hline \multicolumn{4}{|l|}{ Sex } \\
\hline Male & $388(55.0)$ & $48(12.4)$ & $85(21.9)$ \\
\hline Female & $318(45.0)$ & $38(11.9)$ & $31(9.7)$ \\
\hline \multicolumn{4}{|l|}{ Smoking history } \\
\hline Never & $386(54.7)$ & $47(12.2)$ & $4 I(10.6)$ \\
\hline Former/current & $320(45.3)$ & $39(12.2)$ & $75(23.4)$ \\
\hline \multicolumn{4}{|l|}{ ECOG PS } \\
\hline $0-1$ & $538(76.2)$ & $52(9.7)$ & $58(10.8)$ \\
\hline$\geq 2$ & $168(23.8)$ & $34(20.2)$ & $58(34.5)$ \\
\hline \multicolumn{4}{|l|}{ BMI (kg/m2) } \\
\hline$<25$ & $488(69.1)$ & $60(12.3)$ & $86(17.6)$ \\
\hline$\geq 25$ & $218(30.9)$ & $26(11.9)$ & $30(13.8)$ \\
\hline \multicolumn{4}{|l|}{ Tumor histology } \\
\hline Adenocarcinoma & $586(83.0)$ & $76(13.0)$ & $84(14.3)$ \\
\hline Non-adenocarcinoma & $120(17.0)$ & $10(8.3)$ & $32(26.7)$ \\
\hline Squamous cell carcinoma & $106(15.0)$ & $8(7.5)$ & $26(24.5)$ \\
\hline Other NSCLC & $14(2.0)$ & $2(14.3)$ & $6(42.9)$ \\
\hline \multicolumn{4}{|l|}{ Driver oncogene type } \\
\hline EGFR mutated & $306(43.3)$ & $27(8.8)$ & $15(4.9)$ \\
\hline KRAS mutated & $80(11.3)$ & $10(12.5)$ & $24(30.0)$ \\
\hline$A L K$ rearrangement & $36(5.1)$ & $9(25.0)$ & $4(I I . I)$ \\
\hline \multicolumn{4}{|l|}{ Intracranial metastasis } \\
\hline Yes & $102(14.4)$ & II (10.8) & $15(14.7)$ \\
\hline No & $604(85.6)$ & $75(12.4)$ & $104(17.2)$ \\
\hline \multicolumn{4}{|l|}{ Treatment method } \\
\hline Targeted Therapy & $328(46.5)$ & $32(9.8)$ & $14(4.3)$ \\
\hline Without targeted therapy & $378(53.5)$ & $54(14.3)$ & $102(26.9)$ \\
\hline Chemotherapy & $244(34.6)$ & $29(9.5)$ & $45(18.4)$ \\
\hline Other & $134(18.9)$ & $25(34.2)$ & $57(42.5)$ \\
\hline VTE type & $706(100)$ & $86(100)$ & \\
\hline PE alone & $9(1.3)$ & $9(10.4)$ & $4(44.4)$ \\
\hline DVT alone & $59(8.5)$ & $59(68.6)$ & $16(26.7)$ \\
\hline PE and DVT & $18(2.5)$ & $18(20.9)$ & $4(22.2)$ \\
\hline No VTE & $620(87.7)$ & $0(0.0)$ & $92(14.9)$ \\
\hline
\end{tabular}

Abbreviations: NSCLC, non-small cell lung cancer; VTE, venous thromboembolism; ECOG, Eastern Cooperative Oncology Group; PS, performance status; BMI, Body mass index; EGFR, Epidermal growth factor receptor; KRAS, Kirsten rat sarcoma; ALK, anaplastic lymphoma kinase; PE, Pulmonary embolism; DVT, deep vein thrombosis.

the occurrence of VTE and the relationship with early mortality. Our results showed that the risk of VTE occurrence in metastatic NSCLC patients with $A L K$ rearrangements was significantly higher than the risk in $A L K$ wild-type patients. With the approved use of driver oncogene targeted therapies, patients with an EGFR 
Table 2 Univariable and Multivariable Factors Associated with Risk of VTE in Patients with Metastatic NSCLC

\begin{tabular}{|c|c|c|c|c|}
\hline \multirow[t]{2}{*}{ Variables } & \multirow{2}{*}{$\frac{\text { VTE Univariate Analysis }}{p \text { value }}$} & \multicolumn{3}{|c|}{ VTE Multivariate Analysis } \\
\hline & & $p$ value & SHR & $95 \% \mathrm{Cl}$ \\
\hline Age (years) $(\geq 70$ vs $<70)$ & 0.024 & 0.022 & 1.678 & $1.073-2.600$ \\
\hline Sex (male vs female) & 0.894 & - & - & - \\
\hline ECOG PS ( $\geq 2$ vs $0 / 1)$ & $<0.001$ & 0.002 & 1.946 & $1.277-2.970$ \\
\hline Smoker (current/former vs never) & 0.987 & - & - & - \\
\hline BMI $\left(\mathrm{kg} / \mathrm{m}^{2}\right)(\geq 25$ vs $<25)$ & 0.869 & - & - & - \\
\hline \multicolumn{5}{|l|}{ Driver oncogene type } \\
\hline EGFR (mutated vs wild-type) & 0.018 & 0.140 & 0.712 & $0.452-1.120$ \\
\hline KRAS (mutated vs wild-type) & 0.924 & - & - & - \\
\hline ALK (rearrangement vs wild-type) & 0.014 & 0.015 & 2.377 & $1.186-4.760$ \\
\hline
\end{tabular}

Abbreviations: NSCLC, non-small cell lung cancer; VTE, venous thromboembolism; SHR, sub-distribution hazard ratio; ECOG, Eastern Cooperative Oncology Group; PS, performance status; BMI, Body mass index; EGFR, Epidermal growth factor receptor; KRAS, Kirsten rat sarcoma; ALK, anaplastic lymphoma kinase.

mutation/ALK rearrangement had significantly lower rates of early mortality; however, the occurrence of VTE remained an important risk factor for early mortality in metastatic NSCLC patients, especially in those with $E G F R / A L K$ wild-type genes.

\section{Risk Analysis for VTE Age and VTE}

An age of 70 years or older at diagnosis was significantly associated with the occurrence of VTE in our cohort; this finding is consistent with that of a previous report showing that an age greater than 77 years was an independent risk factor for PE in lung cancer patients. ${ }^{18}$ Older patients with metastatic NSCLC are vulnerable to developing VTE, with a higher risk of immobilization and complications like heart and pulmonary disease.

\section{ECOG PS and VTE}

Higher rates of VTE were observed in patients with a poor ECOG PS, which is consistent with the findings of previous research. ${ }^{19,20}$ Patients with a poor PS commonly suffer from more complications than do those with a better PS, and they lose mobility, which increases the risk of VTE occurrence. In this study, we also found that the VTE events were most pronounced in the first 60 days. Even after accounting for competing risk of death and study withdrawal, the increased cumulative risk of VTE remained the highest during the first 60 days. However, it may be that the patients' PS was improved or their tumor burden was reduced after initiating anticancer therapy.

\section{Driver Oncogene Alterations and VTE}

Currently, EGFR, KRAS, and $A L K$ are the most common driver oncogenes tested in patients with metastatic
NSCLC. Our study revealed that the risk of VTE occurrence was 2.377-fold higher in patients exhibiting an $A L K$ rearrangement than in patients with an $A L K$ wild-type gene.

Previous studies ${ }^{7,8,10,21}$ have likewise shown that assessing the $A L K$ status might help determine the risk of VTE among NSCLC subtypes. Therefore, clinicians should pay attention to symptoms of thrombosis in patients exhibiting an $A L K$ rearrangement, both at the time of diagnosis and during follow-up to quickly identify the existence of a VTE event. EGFR mutations, however, were not independent predictors for the occurrence of VTE in this study, which is inconsistent with previous findings demonstrating that $E G F R$ mutations had a negative relationship with VTE risk in patients with NSCLC. ${ }^{9,22}$ Possible explanations for these differential outcomes may be that our study only included stage IV NSCLC patients and that the follow-up period was limited to six months. Thus, the long-term incidence of VTE needs to be explored further.

\section{Other Factors and VTE}

No differences in the occurrence of VTE were observed based on sex or smoking history. Although a BMI $\geq 35 \mathrm{~kg} /$ $\mathrm{m}^{2}$ was reported to be a significant risk factor in previous research, ${ }^{23}$ in this Chinese patient cohort, only five patients had a BMI in this range; therefore, we reduced the cut-off to $\geq 25 \mathrm{~kg} / \mathrm{m}^{2}$. No significant difference in the occurrence of VTE was observed based on the BMI, which is similar to the findings of previous Asian studies. ${ }^{24,25}$

Post hoc analysis of data from a multicenter, prospective trial ${ }^{26}$ demonstrated that anemia was a risk factor for VTE in cancer patients, ${ }^{27}$ and our previous cross-sectional 
A

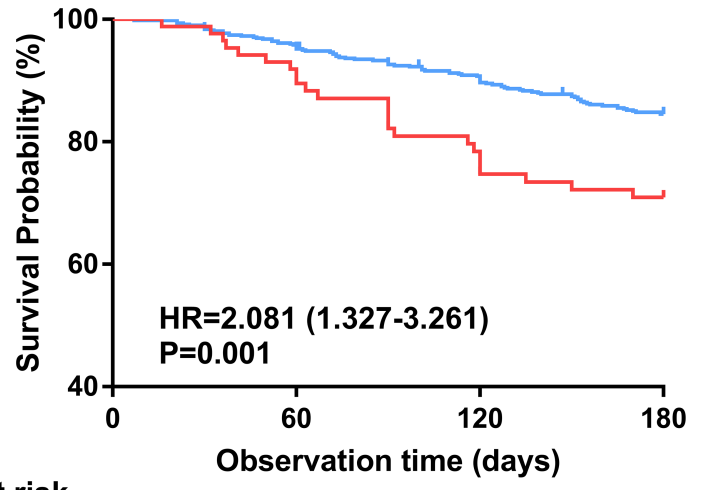

No. at risk

$\begin{array}{rrrr}86 & 79 & 63 & 56 \\ -\quad 620 & 593 & 529 & 488\end{array}$

B

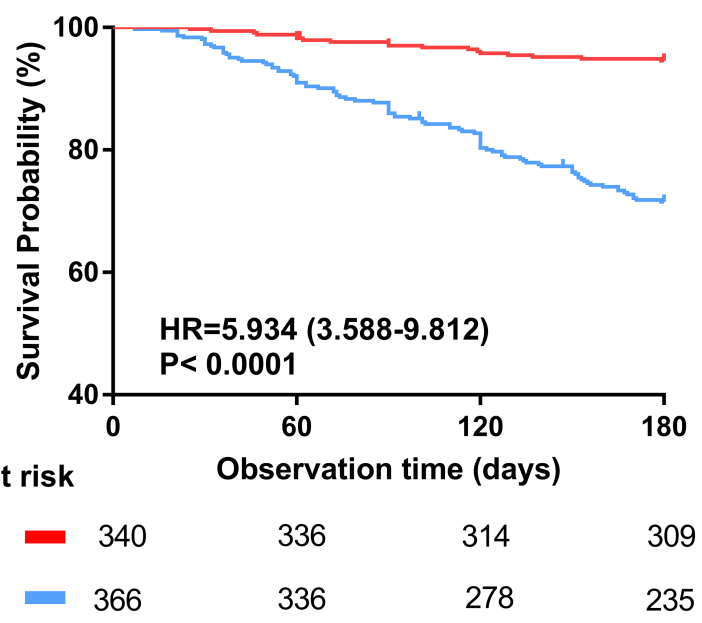

C

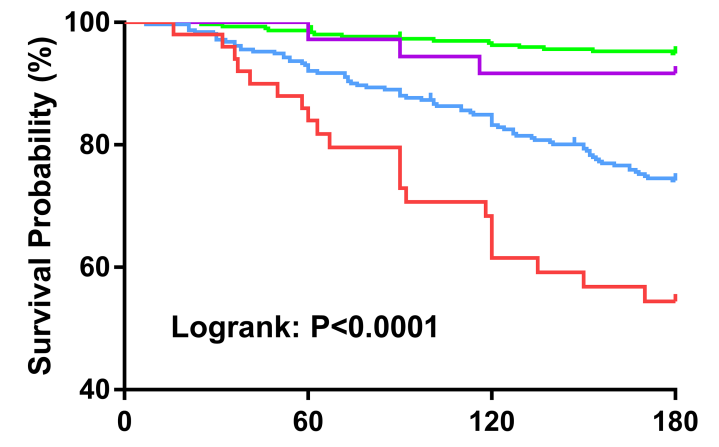

No. at risk

Observation time (days)

$\begin{array}{cccc}50 & 43 & 30 & 23 \\ 316 & 293 & 248 & 212 \\ 304 & 300 & 281 & 276 \\ 36 & 36 & 33 & 33\end{array}$

— With VTE

— Without VTE
- EGFR mutation IALK rearrangement

- EGFR /ALK wild-type
- EGFR /ALK wild-type with VTE

- EGFR IALK wild-type without VTE

- EGFR mutation IALK rearrangement with VTE

- EGFR mutation IALK rearrangement without VTE

Figure 2 The hazard analysis of early mortality. (A) Patients with occurrence of VTE showed significantly associated with early mortality. (B) Patients with EGFR mutation / ALK rearrangement showed a significant protective effect for early mortality. (C) Early mortality among the driver oncogene groups with different VTE status differed significantly using log-rank test $(\mathrm{P}<0.000 \mathrm{I})$.

Abbreviations: EGFR, epidermal growth factor receptor; ALK, anaplastic lymphoma kinase; VTE, venous thromboembolism. 
Table 3 Univariate and Multivariate Cox Regression Analyses of VTE Status with Early Mortality

\begin{tabular}{|c|c|c|c|c|}
\hline \multirow[t]{3}{*}{ Survival Outcomes } & \multicolumn{4}{|c|}{ Early Mortality } \\
\hline & \multicolumn{2}{|c|}{ Univariate Analysis } & \multicolumn{2}{|c|}{ Multivariate Analysis } \\
\hline & $p$ value & HR (95\% Cl) & $p$ value & Adjusted HR (95\% Cl) \\
\hline \multicolumn{5}{|l|}{ Overall cohort $(n=706)$} \\
\hline VTE (yes vs no) & 0.001 & $2.08 \mathrm{I}(1.327-3.26 \mathrm{I})$ & 0.008 & $1.863(1.178-2.947)$ \\
\hline \multicolumn{5}{|l|}{ EGFR mutation/ALK rearrangement $(n=340)$} \\
\hline VTE (yes vs no) & 0.417 & $1.670(0.484-5.770)$ & - & - \\
\hline \multicolumn{5}{|l|}{ EGFR/ALK wild-type $(\mathrm{n}=366)$} \\
\hline VTE (yes vs no) & 0.003 & $2.091(1.290-3.390)$ & 0.041 & $1.682(1.023-2.768)$ \\
\hline
\end{tabular}

Abbreviations: VTE, venous thromboembolism; EGFR, Epidermal growth factor receptor; ALK, anaplastic lymphoma kinase; HR, hazard ratio; Cl, confidence interval.

study also revealed that anemia was related to the occurrence of VTE in lung cancer patients. ${ }^{6}$ In the present study, we also evaluated whether anemia was related to the occurrence of VTE; however, after adjusting for driver oncogene alterations, there was no statistically significant difference between patients with hemoglobin $<10 \mathrm{~g} / \mathrm{dl}$ and $\geq 10 \mathrm{~g} / \mathrm{dl}$. In future studies, we plan to expand the sample size for further validation.

\section{Association Between VTE and Early Mortality}

In our cohort, patients with VTE remained at a remarkably higher risk of early mortality than those without VTE, even after adjusting for other factors like sex, age, BMI, intracranial metastasis, tumor histology, and driver oncogene type (Figure 2A). This is consistent with previously published reports indicating that cancer patients with VTE experienced a higher risk of all-cause mortality than those without VTE during chemotherapy. ${ }^{15,28}$

However, in the modern era of driver oncogene targeted therapy, subgroups of patients with EGFR mutations $/ A L K$ rearrangements experienced significantly improved survival outcomes, and our cohort did show that the risk of early mortality was much higher in patients with $E G F R / A L K$ wild-type genes. The stratified analyses demonstrated that the group with $E G F R$ mutations/ALK rearrangements who did not experience VTE displayed the lowest early mortality rate, whereas the $E G F R / A L K$ wildtype group who experienced VTE displayed the poorest survival, with higher rates of early mortality compared with the former group. We suggest the possibility that actively targeted therapies reduced the tumor burden quickly and improved the hypercoagulable state in these patients, thereby abrogating the risks of VTE on patient survival.

In the $E G F R / A L K$ wild-type subgroup, the patients who experienced VTE exhibited worse survival outcomes compared to those who did not, and the multivariate analysis showed it was a notable predictor of early mortality. It is speculated that several factors may increase the risk of early mortality in these patients. For example, the occurrence of VTE reflected the fact that the hemostatic system becomes activated by carcinoma cells, resulting in cancer progression from a dormant, non-vascularized state toward a metastatic phenotype. ${ }^{11,29,30}$ Besides, the occurrence of VTE itself is associated with a high risk of mortality, such as PE-associated sudden death, except for the side effects of chemotherapy and extra anticoagulation treatment.

\section{Limitations}

This study has several limitations. Firstly, this was a single-center study and was, therefore, subject to potential biases that may have influenced our results. Secondly, not all patients were tested for rare mutations, such as ROS1, and only a few positive ones have been identified in this cohort, so no further analysis was performed. Finally, the prevalence of VTE may have been underestimated, as we did not screen for venous thrombosis in all enrolled patients and some asymptomatic VTE cases might have been overlooked.

\section{Conclusion}

In this prospective, real-world, Chinese cohort study, we confirmed that patients with $A L K$ rearrangements, those with poor ECOG performance, and those older than 70 years of age experienced a higher risk of VTE, and the occurrence of VTE remained a notable predictor of early 
mortality in metastatic NSCLC patients, especially in the $E G F R / A L K$ wild-type subgroup. Clinicians should pay more attention to the occurrence of VTE in metastatic NSCLC patients to ensure timely interventions. Further studies with a goal of reducing early mortality in this subgroup of patients who experience VTE are needed.

\section{Abbreviations}

VTE, venous thromboembolism; NSCLC, non-small cell lung cancer; DVT, deep venous thromboembolism; PE, pulmonary embolism; BMI, body mass index; $E G F R$, epidermal growth factor receptor; $K R A S$, Kirsten rat sarcoma; $A L K$, anaplastic lymphoma kinase; $\mathrm{Cl}$, Confidence interval; ECOG, Eastern Cooperative Oncology Group; PS, performance status; SHR, sub-distribution hazard radio; HR, hazard ratio; TKI, tyrosine kinase inhibitor.

\section{Data Sharing Statement}

All data analyzed during the current study are available from the corresponding author.

\section{Acknowledgments}

The authors greatly appreciate all patients who contributed to this study and thank Yuanhua Yang (MD, $\mathrm{PhD}$ ), Zhanhong Ma (MD), and Lei Zhang (MD) as the members of the review panel who evaluated the VTE events in this study.

\section{Author Contributions}

All authors made substantial contributions to conception and design, acquisition of data, or analysis and interpretation of data; took part in drafting the article or revising it critically for important intellectual content; agreed to submit to the current journal; gave final approval of the version to be published; and agree to be accountable for all aspects of the work.

\section{Funding}

This research was supported by the National Natural Science Foundation of China (grant number 31770961 and 31570890).

\section{Disclosure}

The authors have no conflicts of interest to declare.

\section{References}

1. Khorana AA, Francis CW, Culakova E, Kuderer NM, Lyman GH. Thromboembolism is a leading cause of death in cancer patients receiving outpatient chemotherapy. $J$ Thromb Haemost. 2007;5 (3):632-634. doi:10.1111/j.1538-7836.2007.02374.x

2. Ruiz-Artacho P, Trujillo-Santos J, Lopez-Jimenez L, et al. Clinical characteristics and outcomes of patients with lung cancer and venous thromboembolism. TH Open. 2018;2(2):e210-e217. doi:10.1055/ s-0038-1656542

3. Walker AJ, Card TR, West J, Crooks C, Grainge MJ. Incidence of venous thromboembolism in patients with cancer - A cohort study using linked United Kingdom databases. Eur J Cancer. 2013;49 (6):1404-1413.

4. Ay C, Pabinger I, Cohen AT. Cancer-associated venous thromboembolism: burden, mechanisms, and management. Thromb Haemost. 2017;117(2):219-230.

5. Blix K, Gran OV, Severinsen MT, et al. Impact of time since diagnosis and mortality rate on cancer-associated venous thromboembolism: the Scandinavian Thrombosis and Cancer (STAC) cohort. J Thromb Haemost. 2018;16(7):1327-1335.

6. Zhang YH, Yang YH, Chen WH, et al. Prevalence and Associations of VTE in patients with newly diagnosed lung cancer. Chest. 2014;146(3):650-658.

7. Zer A, Moskovitz M, Hwang DM, et al. ALK-Rearranged non-smallcell lung cancer is associated with a high rate of venous thromboembolism. Clin Lung Cancer. 2017;18(2):156-161.

8. Al-Samkari H, Leiva O, Dagogo-Jack I, et al. Impact of ALK rearrangement on venous and arterial thrombotic risk in NSCLC. J Thorac Oncol. 2020;15(9):1497-1506.

9. Dou FF, Li HQ, Zhu M, et al. Association between oncogenic status and risk of venous thromboembolism in patients with non-small cell lung cancer. Resp Res. 2018;19(1):88

10. Dou FF, Zhang Y, Yi JW, et al. Association of ALK rearrangement and risk of venous thromboembolism in patients with non-small cell lung cancer: a prospective cohort study. Thromb Res. 2020;186:36-41. doi:10.1016/j.thromres.2019.12.009

11. Kuderer NM, Poniewierski MS, Culakova E, et al. Predictors of venous thromboembolism and early mortality in lung cancer: results from a global prospective study (CANTARISK). Oncologist. 2018;23 (2):247-255. doi:10.1634/theoncologist.2017-0205

12. Siegel RL, Miller KD, Jemal A. Cancer statistics, 2020. CA Cancer J Clin. 2020;70(1):7-30. doi:10.3322/caac.21590

13. Arbour K, Riely G. Systemic therapy for locally advanced and metastatic non-small cell lung cancer: a review. JAMA. 2019;322 (8):764-774. doi:10.1001/jama.2019.11058

14. Howlader N, Forjaz G, Mooradian MJ, et al. The effect of advances in lung-cancer treatment on population mortality. $N$ Engl J Med. 2020;383(7):640-649. doi:10.1056/NEJMoa1916623

15. Khorana AA. Venous thromboembolism and prognosis in cancer. Thromb Res. 2010;125(6):490-493. doi:10.1016/j.thromres.2009.12.023

16. Kourelis TV, Wysokinska EM, Wang Y, Yang P, Mansfield AS, Tafur AJ. Early venous thromboembolic events are associated with worse prognosis in patients with lung cancer. Lung Cancer. 2014;86 (3):358-362. doi:10.1016/j.lungcan.2014.10.003

17. Giroux D, Van Schil P, Asamura H, et al. The IASLC lung cancer staging project: a renewed call to participation. J Thoracic Oncol. 2018;13(6):801-809. doi:10.1016/j.jtho.2018.02.012

18. Liu JJ, Wang P, Yan Y, Song K. Prognosis and risk factors in older patients with lung cancer and pulmonary embolism: a propensity score matching analysis. Sci Rep-Uk. 2020;10(1).

19. Wang Z, Yan HH, Yang JJ, et al. Venous thromboembolism risk factors in Chinese non-small cell lung cancer patients. Support Care Cancer. 2015;23(3):635-641. doi:10.1007/s00520-014$2405-\mathrm{y}$ 
20. Vergati M, Della-Morte D, Ferroni P, et al. Increased risk of chemotherapy-associated venous thromboembolism in elderly patients with cancer. Rejuvenation Res. 2013;16(3):224-231. doi:10.1089/rej.2013.1409

21. Ng TL, Smith DE, Mushtaq R, et al. ROS1 gene rearrangements are associated with an elevated risk of peridiagnosis thromboembolic events. J Thorac Oncol. 2019;14(4):596-605. doi:10.1016/j. jtho.2018.12.001

22. Yu JL, May L, Lhotak V, et al. Oncogenic events regulate tissue factor expression in colorectal cancer cells: implications for tumor progression and angiogenesis. Blood. 2005;105(4):1734-1741.

23. Parkin L, Sweetland S, Balkwill A, Green J, Reeves G, Beral V. Body mass index, surgery, and risk of venous thromboembolism in middle-aged women: a cohort study. Circulation. 2012;125 (15):1897-1904.

24. Lee YG, Kim I, Lee E, et al. Risk factors and prognostic impact of venous thromboembolism in Asian patients with non-small cell lung cancer. Thromb Haemost. 2014;111(6):1112-1120.

25. Chen JS, Hung CY, Chang H, et al. Venous thromboembolism in asian patients with pancreatic cancer following palliative chemotherapy: low incidence but a negative prognosticator for those with early onset. Cancers (Basel). 2018;10(12):501.
26. Henry DH, Dahl NV, Auerbach M, Tchekmedyian S, Laufman LR. Intravenous ferric gluconate significantly improves response to epoetin alfa versus oral iron or no iron in anemic patients with cancer receiving chemotherapy. Oncologist. 2007;12(2):231-242.

27. Henry DH, Dahl NV, Auerbach MA. Thrombocytosis and venous thromboembolism in cancer patients with chemotherapy induced anemia may be related to ESA induced iron restricted erythropoiesis and reversed by administration of IV iron. Am J Hematol. 2012;87 (3):308-310.

28. Hicks LK, Cheung MC, Ding K, et al. Venous thromboembolism and nonsmall cell lung cancer: a pooled analysis of National Cancer Institute of Canada Clinical Trials Group trials. Cancer. 2009;115 (23):5516-5525.

29. Mego M, Karaba M, Minarik G, et al. Relationship between circulating tumor cells, blood coagulation, and urokinase-plasminogenactivator system in early breast cancer patients. Breast J. 2015;21 (2):155-160.

30. Mandoj C, Tomao L, Conti L. Coagulation in brain tumors: biological basis and clinical implications. Front Neurol. 2019;10:181.

\section{Publish your work in this journal}

Cancer Management and Research is an international, peer-reviewed open access journal focusing on cancer research and the optimal use of preventative and integrated treatment interventions to achieve improved outcomes, enhanced survival and quality of life for the cancer patient.
The manuscript management system is completely online and includes a very quick and fair peer-review system, which is all easy to use. Visit http://www.dovepress.com/testimonials.php to read real quotes from published authors. 